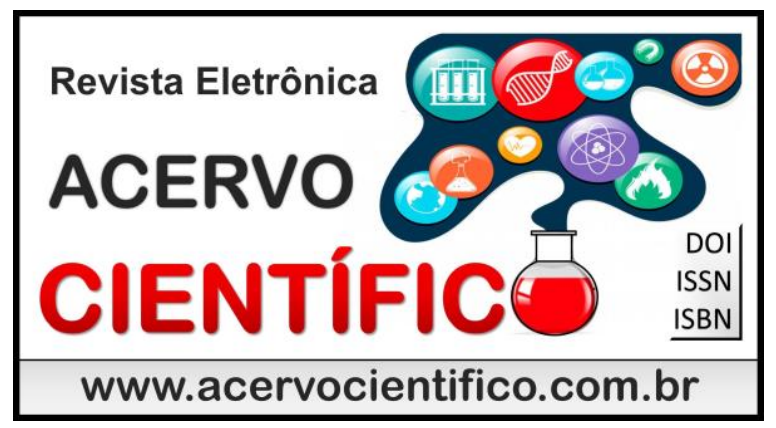

ARTIGO ORIGINAL

Recebido em: 1/2019

Aceito em: 2/2019

Publicado em: 6/2019

\title{
Comparativo do tempo de usinagem em função da estratégia cam utilizada para micro fresamento
}

\author{
Comparative machining time in function of cam strategy used for micro milling \\ Comparación del tiempo de utilización en función de la estrategia de cam usada para \\ micro fresado
}

\section{Wiland Tiergarten Junior ${ }^{1}$}

\begin{abstract}
Resumo: O objetivo deste artigo é apresentar o resultado de pesquisa realizado no setor de moldes e matrizes na área de micro usinagem de micro cavidades. A grande procura por micropeças de alta precisão tem crescido muito em todos os setores, mas principalmente para linha de micro cavidades para indústrias de moldes e matrizes, mas sabe-se que para realizar essas usinagens necessita-se de máquinas de altíssima precisão e rotação. Baseado neste contexto, o objetivo desta pesquisa foi estudar micro usinagens utilizando máquinas não específicas de HSC e nem um software CAM específico para micro usinagem, pois até mesmo nesta tecnologia ainda não existe muitos estudos. Entre tanto a usinagem foi realizada com material Stavax (52HRC) comum. Suas variáveis analisadas foram, tempo de usinagem, numero de linhas do programa CNC e tamanho dos segmentos de reta que cada estratégia obteve, pois o mesmo reflete diretamente no tempo de usinagem. Onde pode-se observar que por mais que a estratégia gerada com o menor numero de linhas, não foi a que menor tempo levou para realizar a usinagem, devido ao seu tamanho de segmento de reta ser menor do que as outra duas estratégias CAM analisadas.
\end{abstract}

Palavras-chave: Máquinas não específicas de HSC. Software CAM. Material Stavax(52HRC). Programas CAM. Segmentos de reta.

\begin{abstract}
The objective of this paper is to present the result of research on the segment in mold and die industry in the area of micro machining for micro cavities. The high demand for high-precision micro parts has been growing in all sectors, but especially in the line of micro cavities in mold and die industries, but it is known that to perform these machining the needs are very high precision and rotating machines. Based on this context, the objective of this research was to study micro machining using non-specific machines for HSC either a specific CAM software for micro machining, even for this technology, it does not exist many studies yet. Between both machining was performed with Stavax (52HRC) material. Its variables analyzed were, machining time, line number of the CNC program and size of line segments that each strategy had, because it reflects directly on the machining time. Whereas it can be seen that while the strategy generated with the smaller number of lines are not less than the time it took to perform the machining, due to the straight segment size is smaller than the other two analyzed CAM strategies.
\end{abstract}

Keywords: non-specific machines HSC. CAM software. Stavax material (52HRC). CAM programs. Line segments.

\footnotetext{
${ }^{1}$ Engenheiro Mecânico da Btomec Ferramentaria e Usinagem de Precisão Ltda, Joinville - SC.

E-mail: wiland t@hotmail.com
} 
Resumen: El objetivo de este artículo es presentar el resultado de investigación realizado en el sector de moldes y matrices en el área de micro mecanizado de micro cavidades. La gran demanda por microperlas de alta precisión ha crecido mucho en todos los sectores, pero principalmente en la línea de micro cavidades para industrias de moldes y matrices, pero se sabe que para realizar esos mecanizados se necesita de máquinas de altísima precisión y rotación. En base a este contexto, el objetivo de esta investigación fue estudiar micro mecanizados utilizando máquinas no específicas de HSC y ni un software CAM específico para micro mecanizado, pues incluso en esta tecnología aún no existen muchos estudios. Entre tanto el mecanizado fue realizado con material Stavax $(52 \mathrm{HRC})$ común. Sus variables analizadas fueron, tiempo de mecanizado, número de líneas del programa CNC y tamaño de los segmentos de recta que cada estrategia obtuvo, pues el mismo refleja directamente en el tiempo de mecanizado. Donde se puede observar que por más que la estrategia generada con el menor número de líneas, no fue la que menor tiempo llevó para realizar el mecanizado, debido a su tamaño de segmento de recta es menor que las otras dos estrategias CAM analizadas.

Palabras clave: Máquinas no específicas de HSC. Software CAM. Material Stavax (52HRC). Programas CAM. Segmentos de recta.

\section{INTRODUÇÃO}

Durante os últimos 20 anos a demanda por micro peças de alta precisão e micro características tem crescido muito em vários setores incluindo aeroespacial, medicinal, automotivo, eletrônico, ótico entre outros. Algumas aplicações bem específicas incluem micro canais para carga de baterias, fluido de micro químico de reatores, micro bicos para injeção de alta temperatura, micro furos e micro cavidade para moldes e matrizes (CHAE et al., 2006).

Para dar suporte há este grande crescimento do mercado de inúmeras aplicações para micro fabricação, existe uma enorme necessidade para crescimento rápido, direto, realizável e que seja possível a repetitividade para fabricar superfícies complexas e de alta precisão.

Pensando nisso, uma alternativa de fabricação para micro mecânica e que está em crescimento é a micro usinagem. É um método novo, mas que sua tecnologia já está bem avançada comparada ao tempo de estudo e tem a capacidade de criar superfícies complexas em escalas muito pequenas como micrométricas e em diferentes materiais (BRECHER et al. 2008).

Além disso, a micro usinagem proporciona inúmeras vantagens, como flexibilidade, custo versus eficiência, repetibilidade e alta precisão de forma. Até então tem uma importante e quase que insubstituível aplicação como; medicinal, implante dental, ponte, próteses, plaquetas para ossos e roscas etc.

1. Mercado de relógios: Caixa de relógios super precisos, peças de movimentos, eletrodos, engrenagens etc.

2. Eletrônicos: Micro moldes para conectores, mini produtos eletrônicos, micro sensores, chips etc.

3. Ótica: Micro lentes, dispositivos óticos etc.

4. Aeroespacial: Moldes para engrenagens, miniaturas para dispositivos, micro válvulas etc.

5. Indústria de moldes em geral.

De uma forma geral micro fresamento, que faz parte do campo da micro usinagem, emerge em um novo método de desenvolvimento, sua forma natural e originaria veio diretamente de uma escala de fresamento convencional. Os dois processos de corte tem os movimentos semelhantes e o processo de corte pode-se ter uma interação com o corte da ferramenta e o tipo de material usinado causando micro quebras (ALTING et al., 2003).

No entanto, micro fresamento tem uma grande importância no crescimento do espaço entre a usinagem tradicional de precisão e a micro usinagem para o mercado de componentes de micro componentes. Contudo, um número de problemas críticos é abordado na usinagem tradicional para micro usinagem, resultando em mecanismos de processos. Seus resultados são mudança na formação de cavaco, forças de corte, vibrações e estabilidade de processo. Essas contradições, por exemplo, ocorre gasto na vida útil e falha prematura imprevisível na ferramenta (LIU et al.,2004).

Com base nestas informações será realizado um estudo teórico/prático relacionado a micro usinagem de cavidades de moldes e matrizes analisando sistema CAM, analisando também as máquinas disponíveis no 
local dos ensaios de usinagem, com principal objetivo de redução de custos através de um conhecimento mais específico de todas as variáveis envolvidas no processo.

Feito este estudo será possível realizar uma análise comparativa de usinagem do aço Stavax com a ferramenta esférica de metal duro de $0,5 \mathrm{~mm}$ de diâmetro com tratamento superficial TiAln (Titanium Aluminium Nitrede) nas máquinas Mikron VCP 800 e Mikron VCE 800 Pro com o softwares Power Mill.

Esse processo de usinagem será executado nas máquinas Mikron VCP 800 e Mikron VCE 800 Pro com o software Power Mill.

Micro fresamento não é um novo tipo de usinagem, é simplesmente uma escala menor de uma usinagem de tamanho comum. Na verdade, segundo alguns estudos a definição de micro fresamento é fresas menores que um milímetro de diâmetro.

Por isso, basicamente a sistemática de movimentos da micro usinagem são as mesmas que de uma usinagem de tamanho normal. Os três principais componentes para uma micro usinagem são: ferramenta de corte, máquina ferramenta e a peça (TLUSTY., 2000).

a. Ferramenta de corte: Micro fresas comerciais tem a geometria similar as fresas de tamanho "normal". O substrato do material comercializado é basicamente os de grão finos (grão menores que 0,5 micras) metal duro e tungstênio com cobalto, sendo o revestimento mais comum, que será mais bem explicado ao longo do trabalho é o TiAIN. As micros fresas são a maioria das vezes fabricadas pelos métodos de retificação, pois a qualidade do micro corte da fresa depende da propriedade do rebolo da retifica, assim como o tamanho do rebolo, raio e desgaste. Com a contínua pesquisa e desenvolvimento de ferramentas, hoje já é possível fabricar por retificação fresas com diâmetros de 0,025 milímetros (TORRES et al., 2009).

b. Máquina ferramenta: No início das etapas de pesquisa de micro usinagem, as pesquisas experimentais foram realizadas em máquinas comuns, de macro usinagem. Com o crescimento do entendimento do processo, foi cada dia mais requerido à pesquisa dessa tecnologia, assim como empresas de máquinas. Suas perguntas eram sobre rigidez, amortecimento, deformação térmica, velocidade do spindle e aceleração (ADAMS et al., 2001). Devido a isso máquinas específicas para micro usinagem são encontradas cada dia mais em literaturas.

c. Peça: Uma grande gama de material pode ser usinada pelo processo de micro usinagem, assim como aluminum até aço de liga. Essas pesquisas todas estão sendo realizadas devido à grande vantagem que a micro usinagem com ferramentas de corte pode fazer na substituição de processos como EDM.

Qualidade e capacidade ótima da máquina ferramenta são vitais para produção de micro produtos, para alcançar estes requisitos de alta precisão, boa forma e ótima qualidade superficial. As máquinas de micro fresamento devem ter alta rigidez, estabilidade térmica, baixo erros de movimentos e um alto amortecimento ou dinamismo de rigidez (HUO, 2009).

Somando a isso, uma alta velocidade de corte necessita atingir uma performance muito boa da ferramenta e um alto índice de remoção de material. Por causa de dimensões pequenas para micros ferramentas, máquinas para micro usinagem são normalmente equipadas com alta rotação e baixa expansão térmica (CHAE et al., 2006).

Segundo as empresas Kern, Mikron, Makino e Röders (2014) no início do desenvolvimento da micro usinagem, as linhas de fresamento eram principalmente alcançadas com máquinas convencionais, nas quais não tinham custo efetivo e tinham pouca flexibilidade. Ao longo do crescimento da compreensão do processo de corte, os requerimentos especiais para as máquinas ferramentas ultra precisas foram configuradas e projetadas com capacidade para micro fresamento.

Outro fator muito importante fará o sucesso da fabricação de micro cavidades com rapidez e precisão está na diferença entre programas CAM, deve ser a flexibilidade e a escolha do caminho da ferramenta de corte. Existe uma gama enorme de estratégias de programa CAM, que podem ser escolhidos, mas esta escolha afeta diretamente na velocidade e na qualidade de acabamento superficial. 
É importante salientar que a maioria dos programas CAM abordam o problema com uma hipótese, que múltipla estratégia de usinagem deve ser usada para proporcionar a máxima eficiência de usinagem (SMITH, 2009).

\section{RESULTADOS E DISCUSSÕES}

\section{Comparativo De Tempo De Usinagem Em Função Da Estratégia Cam}

Para analise da proposta, utilizaram-se quatro diferentes estratégias, elas foram: 1) Passe paralelo; 2) Espiral, 3) Espiral Otimizado; e 4) 3D Offset.

Visto que a literatura e a prática realizada na indústria de moldes e matrizes essas são as estratégias mais utilizadas, mas não é sabido se para micro usinagem seria o mesmo comportamento. Outro fator muito relevante é que todas as trajetórias foram calculadas com interpolação circular/linear, pois a mesma é mais recomendada para esse tipo de usinagem de superfície complexa.

\section{Sistema Cam}

Com O Modelo Matemático Gerado Por Um Software CAD, Foram Realizados Programas NC Usando O Software CAM. Sistema Cam Analisado - Power Mill versão 10.0.06 - Sistema desenvolvido pela empresa Delcam. O mesmo será identificado por software "A". O programa "A" foi disponibilizado pela empresa onde foi realizado o experimento.

\section{Máquina Cnc}

Foram realizados os testes em duas máquinas distintas, Mikron VCP 800 e Mikron VCE 800 Pro ambas se localizam na Btomec Ferramentaria e Usinagem de Precisão Ltda. Elas estão sendo tratadas como máquina " $A$ " e "B" respectivamente ao longo do estudo.

A máquina "A", não é considerada como uma high speed machine, mas tem alguns de seus opcionais HSM, como Spindle com sistema de fixação HSK63 e rotação de 20.000RPM, dimensão de sua mesa X, Y e Z respectivamente $800 \times 450 \times 450 \mathrm{~mm}$, movimentos programados de $25 \mathrm{~m} / \mathrm{min}$. e tolerância de posicionamento conforme a DIN 203-2 é de $0,008 \mathrm{~mm}$. Nesta máquina existe um ajustador térmico de temperatura que conforme há dilatação nos eixos $\mathrm{X}, \mathrm{Y}$ e $\mathrm{Z}$ o sistema corrige a diferença alterando os parâmetros da máquina.

A máquina " $B$ ", é considera uma máquina standard, a dimensão de sua mesa $X, Y$ e $Z$ respectivamente $800 \times 500 \times 540 \mathrm{~mm}$, velocidade de rotação do Spindle é 14.000RPM e sistema de fixação ISO40, movimentos programados de $24 \mathrm{~m} / \mathrm{min}$. e tolerância de posicionamento conforme a DIN 203-2 é de $0,008 \mathrm{~mm}$, mas não possui o sistema de compensação térmica.

\section{Corpo De Prova}

Este material é conhecido como Stavax e é usado em moldes de injeção de plástico, ele é temperado com 52HRC. Foi realizada a preparação de 10 corpos de prova de dimensões $X, Y$ e Z $30 \times 30 \times 10 \mathrm{~mm}$ respectivamente.

\section{Tamanho Dos Programas Cnc}

Notou-se inicialmente que houve variações nos tamanhos das diferentes estratégias CAM, onde que automaticamente está ligado com os números de pontos que o CAM gerou nas suas usinagens na geometria que foi analisada. A figura 01 mostra os resultados.

Percebe-se claramente que a estratégia "espiral otimizado" é a que obteve o menor número de linhas no programa CNC. As outras estratégias apresentaram um número mais elevado de linhas comparando com "espiral otimizada", como 3D-Offset que chegou apresentar até $269 \%$ mais linhas do que o inicial mencionado aqui. 
Figura 01 - Análise do número de linhas.

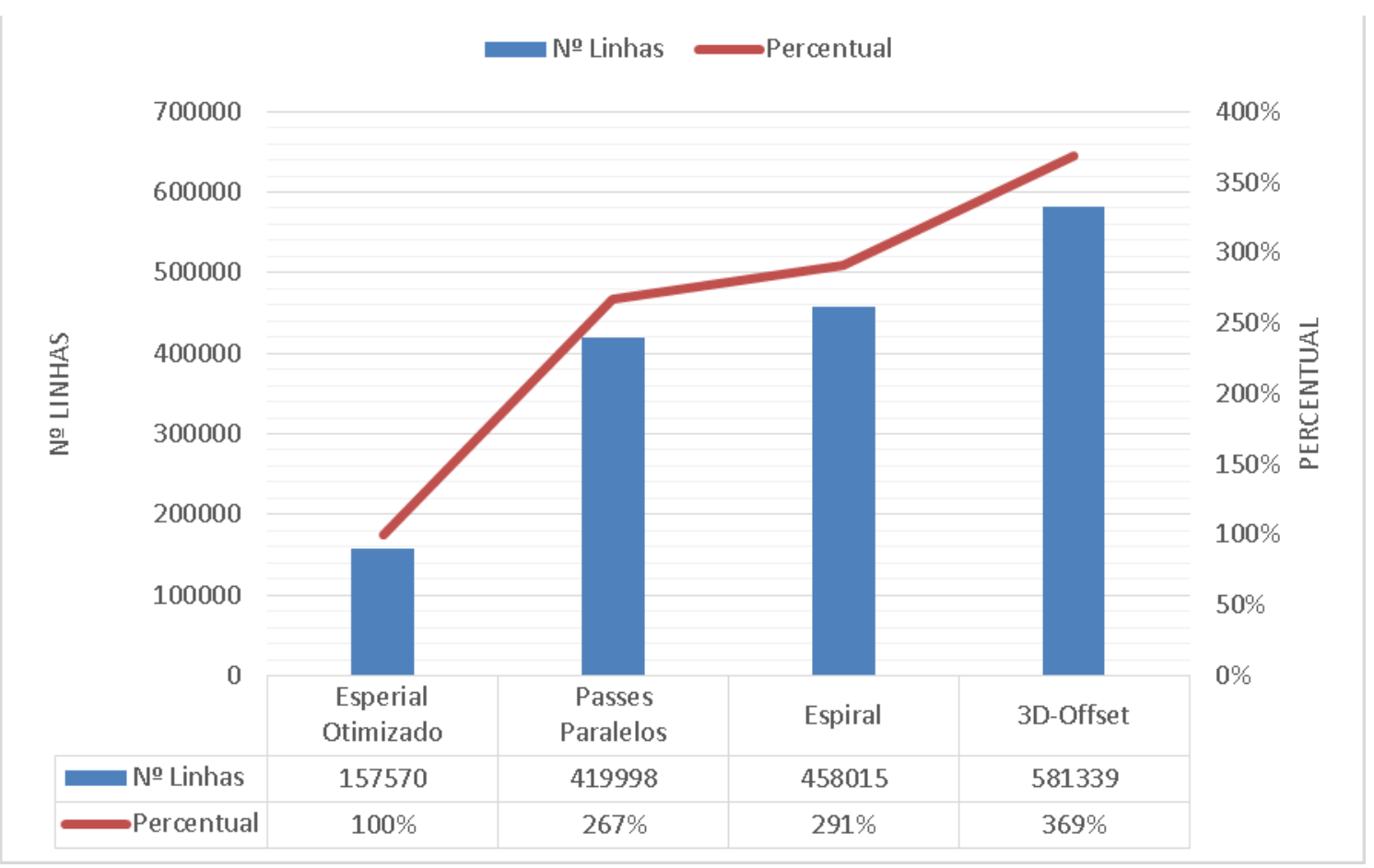

Fonte: Dados obtidos por pesquisa direta.

\section{Tempo De Usinagem}

O tempo de usinagem foi coletado das máquinas "A" e "B", assim como o tempo de usinagem simulado pelo sistema CAM.

Nestas usinagens foi observado, como mostra a figura 02 e a figura 03, uma variação significativa de cada estratégia CAM em relação ao tempo real de usinagem.

Conforme mostra a figura 02, pode-se verificar que a estratégia "Passes Paralelos" apresentou o menor tempo de usinagem. Mas ao mesmo tempo, não é a estratégia que tem o menor número de linhas em seu programa como mostrado anteriormente na figura 01.

Todas essas variações de tempo e tamanho de linha deve-se a dispersão dos pontos ao longo da trajetória do corpo de prova. A estratégia "espiral otimizada" é a que menor apresentou linhas, mas foi a usinagem que mais levou tempo. Já a estratégia "passes paralelos" apresentou maior número de linhas e o melhor tempo de usinagem. Pois a mesma apresentou melhor dispersão dos pontos ao longo da peça.

Em uma análise de melhor visualização, na figura 04, pode-se observar essa diferença de número de linhas e tempo encontrados nos testes.

Tendo em vista que essa variação de tamanho do programa e tempo de usinagem não foi proporcional, foi analisada a média de avanço do programa que efetivamente foi percorrido nas usinagens, como mostra a figura 05.

Como as estratégias "espiral" e "passes paralelos" apresentaram uma diferença de tempo não maior que $2 \%$ entre si, tanto na máquina "A" e na "B". Pode-se observar essa mesma diferença na media de avanço real das mesmas como mostra figura 05.

Se for levar em consideração qual das estratégias escolher para o trabalho entre a "espiral" e a "passes paralelos", ficaria muito difícil. Conforme essas condições, foi analisado o acabamento superficial das duas peças. 
Figura 02 - Tempos obtidos nas diferentes estratégias CAM na máquina "A".

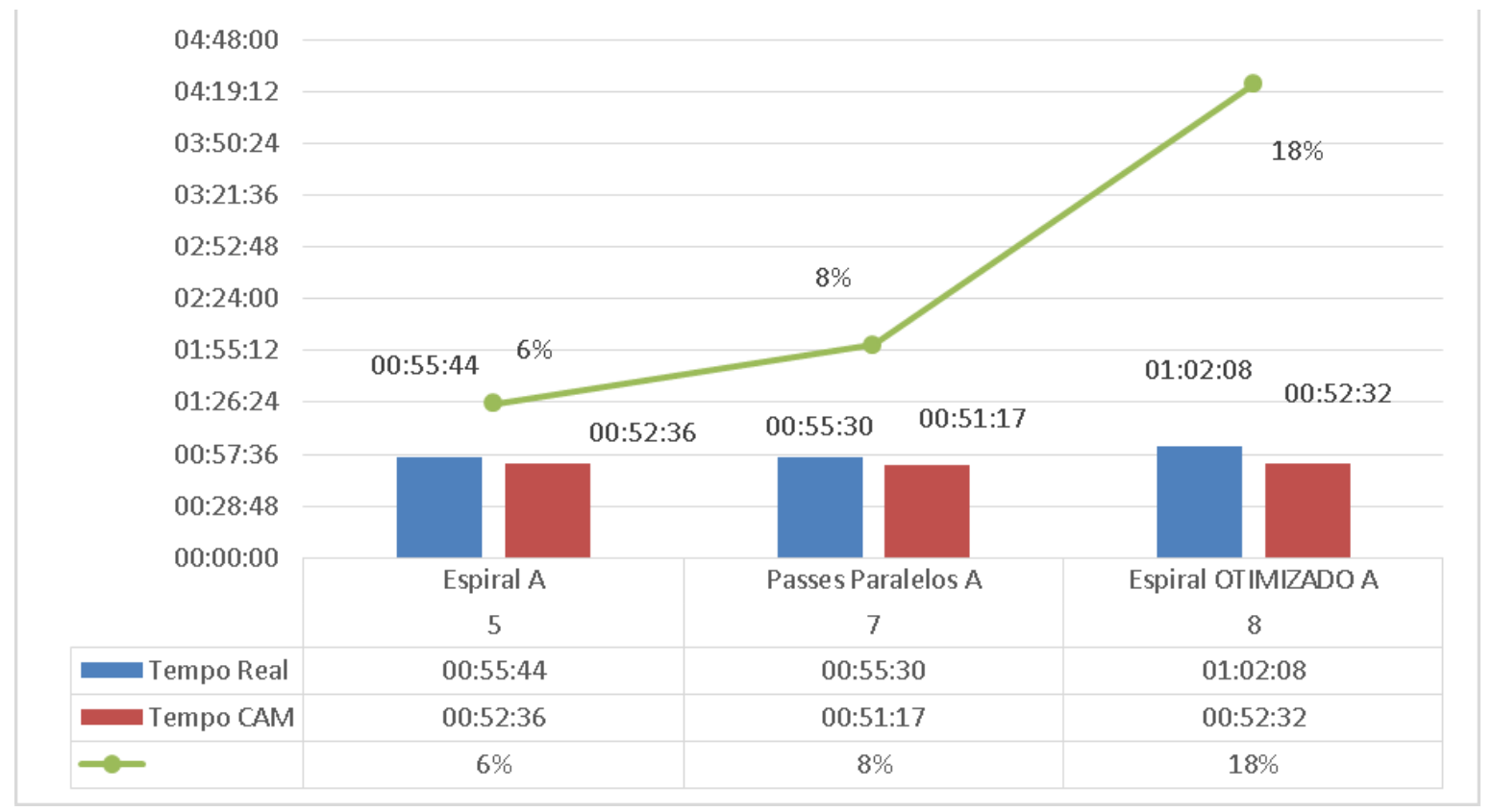

Fonte: Dados obtidos por pesquisa direta.

Figura 03 - Tempos obtidos nas diferentes estratégias CAM na máquina "B".

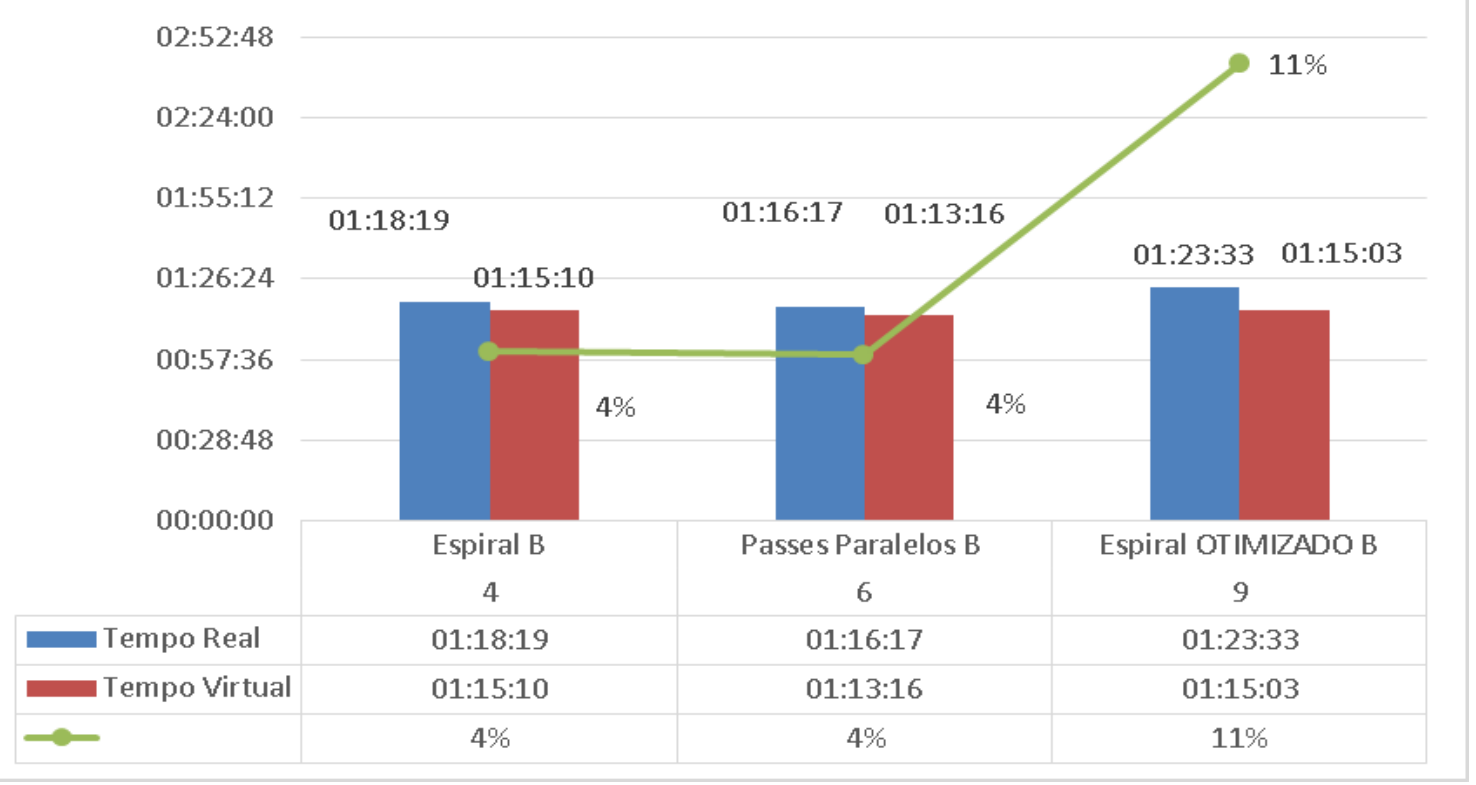

Fonte: Dados obtidos por pesquisa direta. 
Figura 04 - Variação de tamanho de programa CNC por tempo real de usinagem.

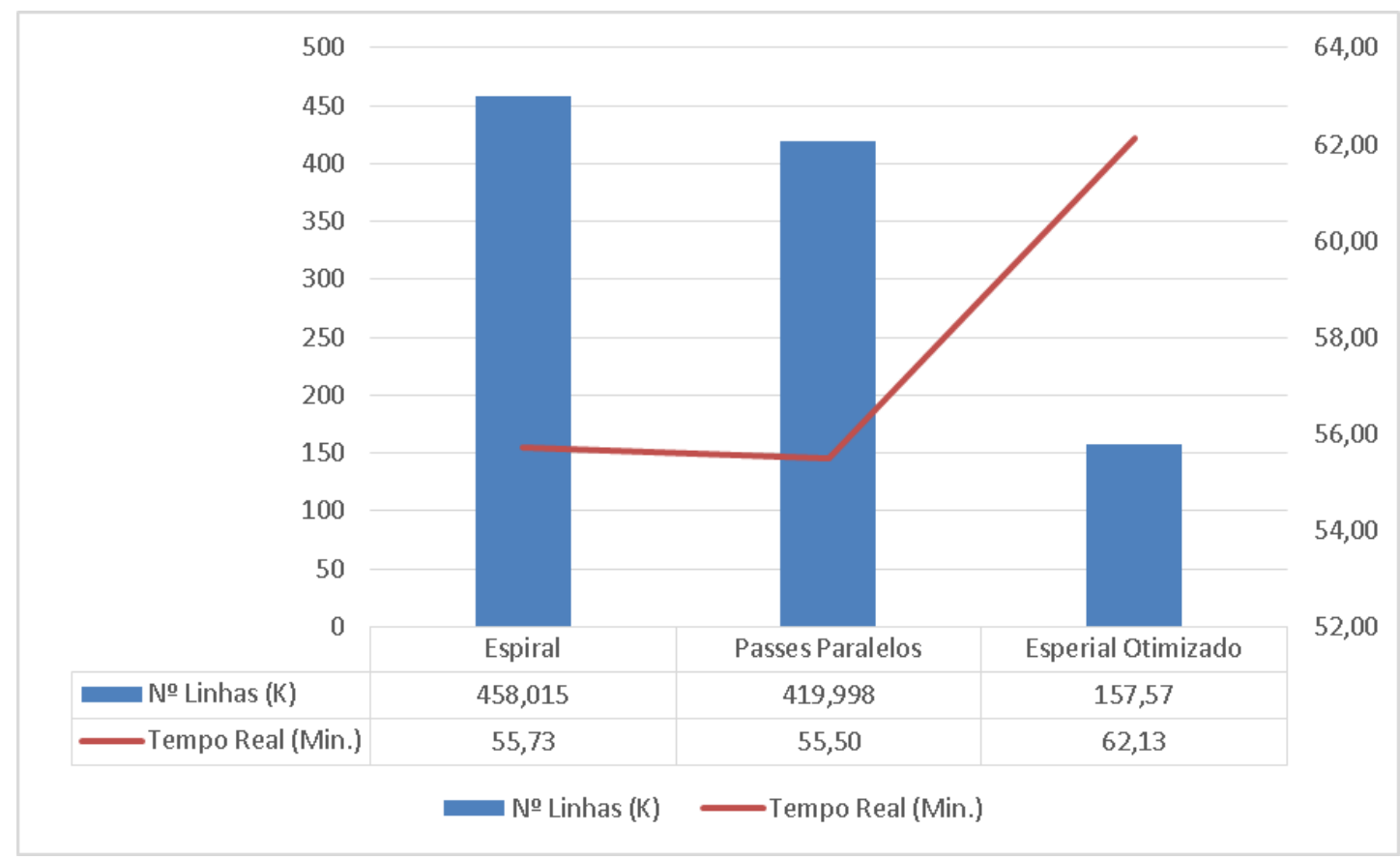

Fonte: Dados obtidos por pesquisa direta.

Figura 05 - Media de avanço efetivo nas usinagens.

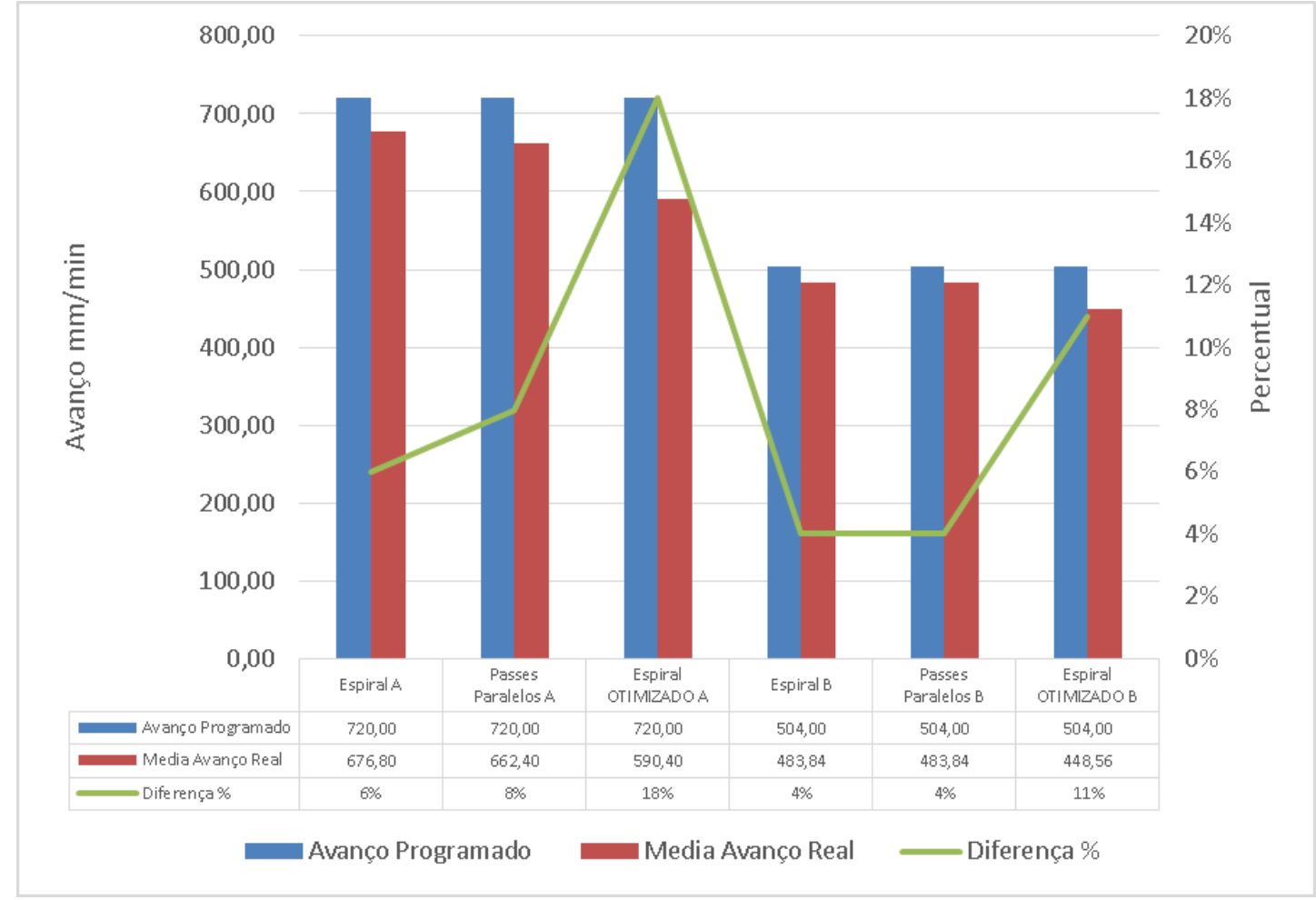

Fonte: Dados obtidos por pesquisa direta. 


\section{Tamanho Do Segmento De Reta}

A figura 06 mostra a variação e a média do segmento de reta gerado pelas diferentes estratégias de usinagem.

Figura 06 Média e Desvio padrão de segmento de reta.

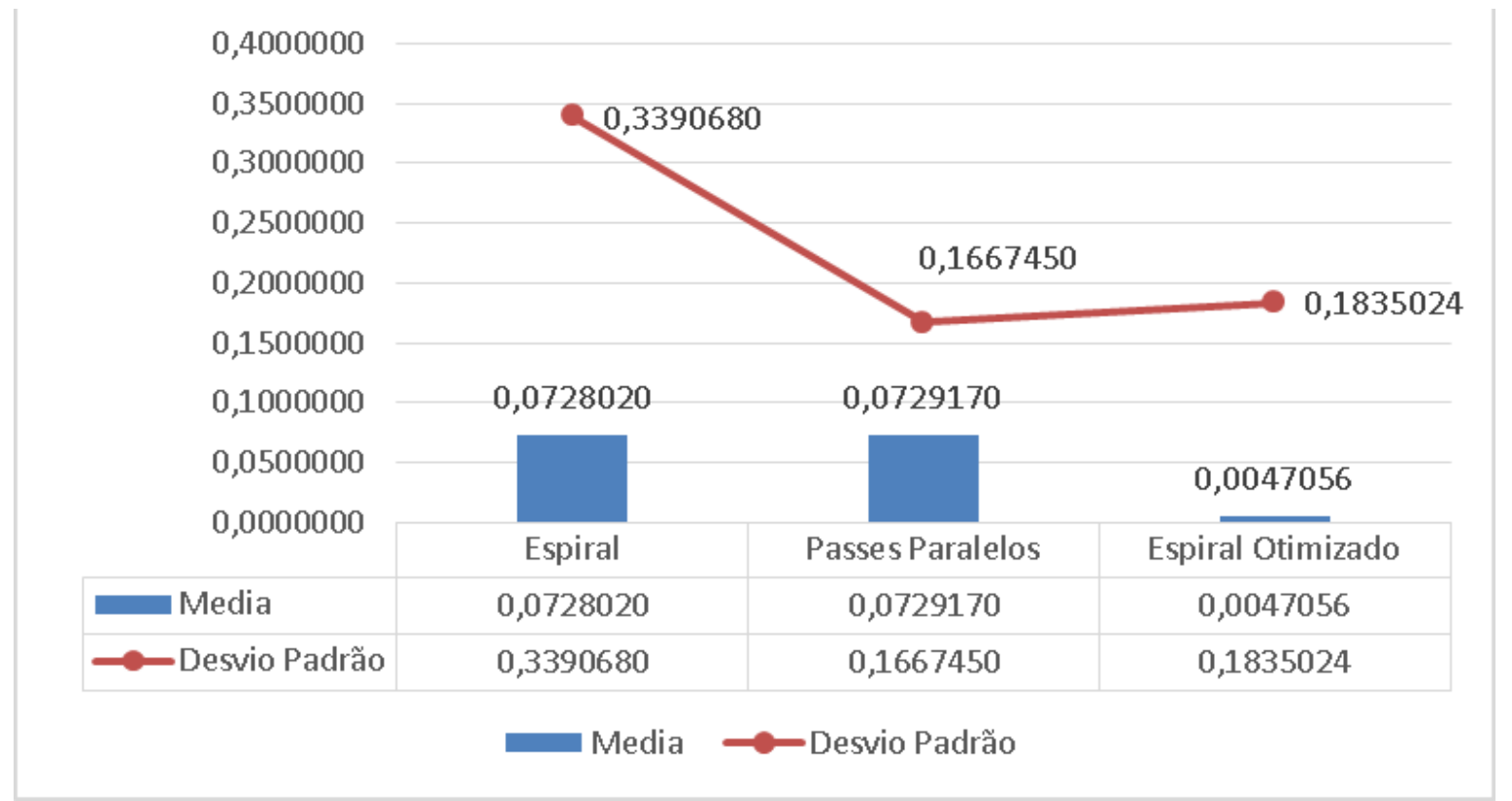

Fonte: Dados obtidos por pesquisa direta.

A estratégia "passes paralelos" foi a usinagem que apresentou a maior media do segmento de reta e o menor desvio padrão. Já a estratégia "espiral" apresentou a segunda maior media do tamanho de segmento de reta, porém apresentou o maior desvio-padrão.

Na estratégia "espiral otimizado" apresentou o menor valor de media do tamanho do segmento de reta, e segundo menor valor em seu desvio-padrão.

Pode-se definir nesta análise, que a melhor estratégia deve-se aos "passes paralelos", pois o mesmo apresentou desvio-padrão mais baixo e o segundo melhor tempo, salientado que a diferença de tempo entre o primeiro melhor tempo não foi maior que $0,4 \%$ na máquina " $A$ ". Onde sua media de avanço na máquina " $A$ " apresentou $-8 \%$ de avanço programado e na máquina " $B$ " $-4 \%$. E nem uma diferença de media de avanço entre a estratégia "passes paralelos" e "espiral" na máquina "B" e $2 \%$ na máquina "A".

$\mathrm{Na}$ figura 07 e figura 08 apresenta o resultado da máquina "A" e "B" comparando o tamanho do segmento de reta pelo tempo, pois na teoria é sabido que quanto menor o tamanho do programa, mais próximo do tempo programado é. Pois o avanço permanece por mais tempo, no avanço programado.

Mas pode-se observar que analisando a estratégia espiral otimizado, onde o programa apresenta 0 menor tamanho, é a estratégia que maior apresenta o tempo. Isso deve-se ao tamanho de segmento de reta, pois o mesmo foi que menor apresentou o segmento de reta, chegando a menos que $0,005 \mathrm{~mm}$, resultado automaticamente em uma redução no avanço da máquina e assim maior tempo de usinagem. 
Figura 07 - Gráfico tamanho segmento de reta por tempo.

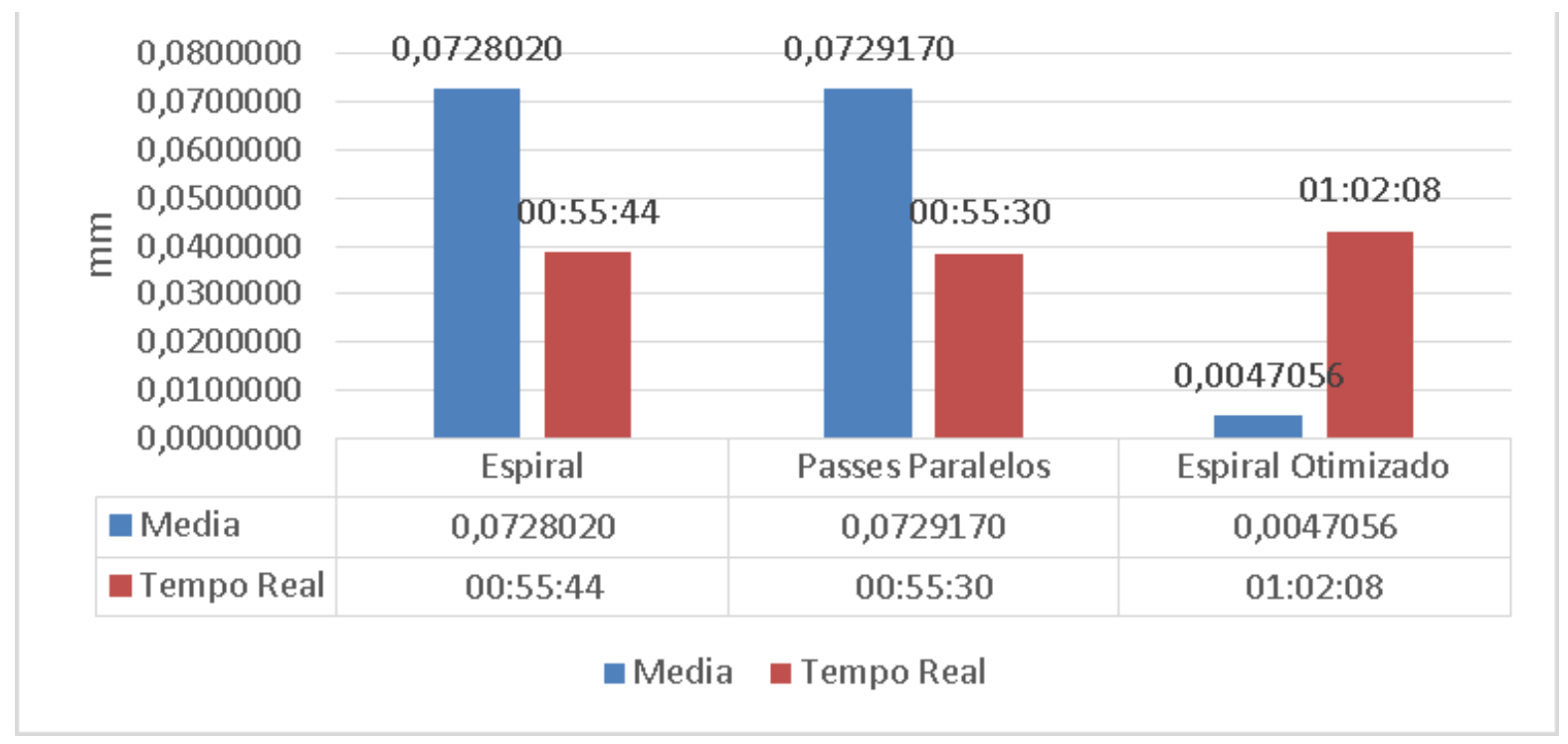

Fonte: Dados obtidos por pesquisa direta.

A figura 08 apresenta a análise da máquina "B". Que apresenta uma grande diferença de tempo de usinagem, mas proporcional ao calculado.

Figura 08 - Gráfico tamanho segmento de reta por tempo.

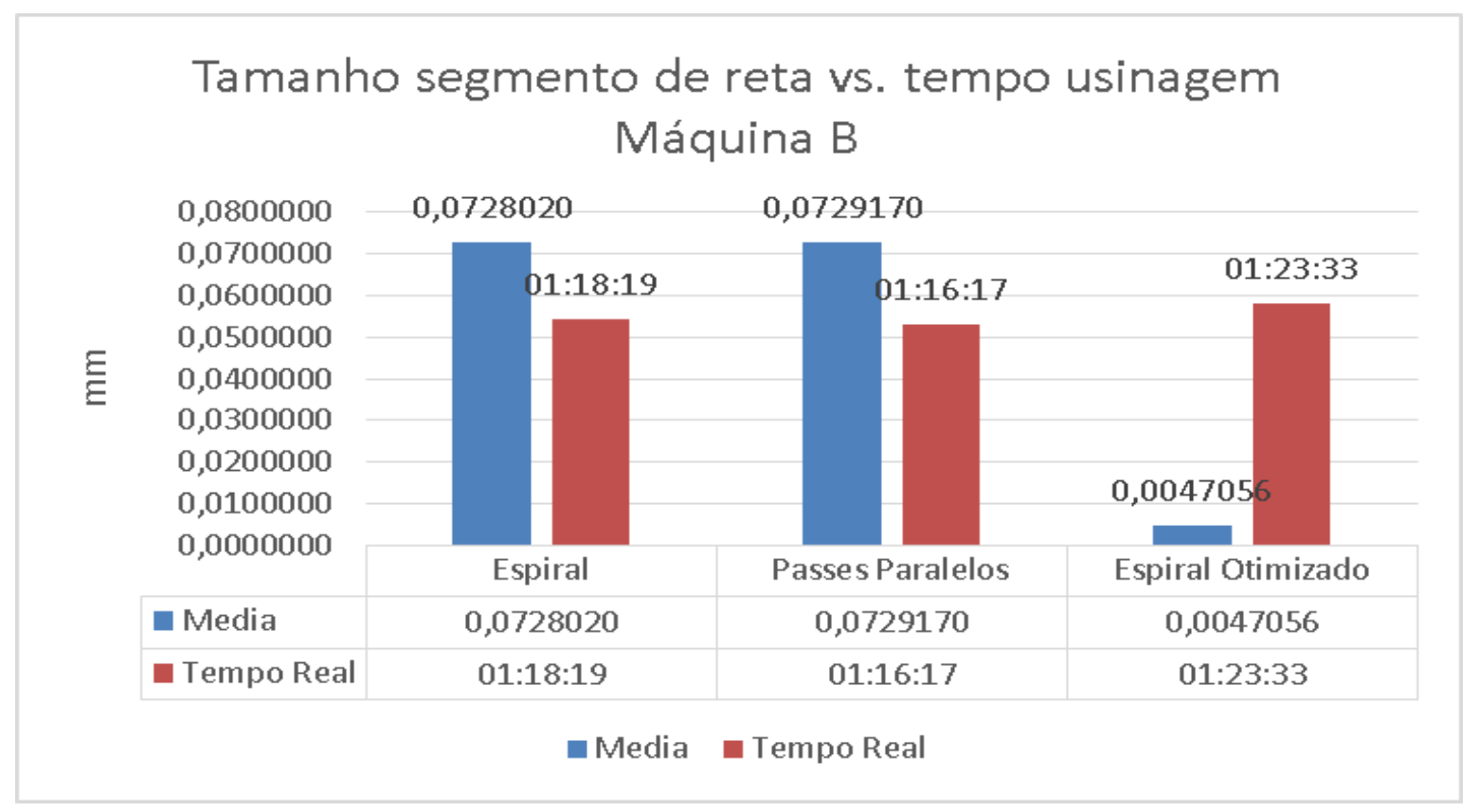

Fonte: Dados obtidos por pesquisa direta. 


\section{CONSIDERAÇÕES FINAIS}

Todas as usinagens e análise experimental conduziram as algumas conclusões:

1. Cada estratégia CAM gerou diferentes números de linhas para realizar a usinagem do mesmo corpo de prova. Essas variações chegaram até $269 \%$ a mais. Isso tudo indica que o mesmo software usa distintos algoritmos matemáticos para realização de diferentes estratégias .Pode-se notar que existe uma ligação entre os tamanhos dos programas NC para o tempo real e simulado de usinagem. Quanto menor segmento de reta, maior é a distancia de avanço programado versus o real. Pois o mesmo reduz constantemente para seguir corretamente os pontos.

2. Nas duas fresadoras CNC apresentaram uma diferença não maior que $2 \%$ entre si nas estratégias de tempo versus tempo real de usinagem.

3. Para máquina "A" usando a estratégia "passes paralelos" onde apresentou menor tamanho de segmento de reta. Foi a máquina que melhor apresentou seu corpo de prova, melhor precisão na geometria da peça, onde seu desvio padrão na geometria foi de $0,0099 \mathrm{~mm}$.

4. Entretanto a máquina "B" apresentou sua melhor usinagem em precisão com a estratégia "espiral" tendo seu desvio padrão em $0,0146 \mathrm{~mm}$

\section{AGRADECIMENTOS E FINANCIAMENTO}

Grato a empresa Btomec Ferramentaria e Usinagem de Precisão por seder seus mais modernas maquinas de fresamento e equipamentos de medição para realizar este estudo que tanto é importante para a industria de manufatura de molde de scala micrometrica.

\section{REFERÊNCIAS}

1. ADAMS, D. P et al. Micromilling of metal alloys with focused ion beam-fabricated tools. 2001.

2. ALTING, L. et al. Micro engineering. CIRP Annals - Manufacturing Technology. 2003.

3. BRECHER, C. et al. Characterization and optimization of the dynamic tool path of a highly dynamic micromilling machine. CIRP Journal of Manufacturing Science and Technology. 2008.

4. CHAE, J. et al. Investigation of micro-cutting operations. International Journal of Machine Tools and Manufacture. 2006.

5. DAGNALL, H. Exploring Surface Texture. 1. ed. IL: Rank Taylor Hobson, 1980.

6. FERRARESI, D. Fundamentos da usinagem dos metais. 9. ed. São Paulo: Edgard Blücher, 1995.

7. SCHNEIDER, G. J. Machinability of metals. American Machinist. 2009.

8. HELLENO, A. L. E SCHÜTZER, K. Programção e transmissão de dados na tecnologia HSC. Usinagem em altíssimas velocidades: como os conceitos HSM/HSC podem revolucionar a indústria metal-mecanica. 2003.

9. HUO, D. at al. Design of five-axis ultra-precision micro-milling machine-UltraMill. Part 1: holistic design approach, design considerations and specifications. International Journal of Advanced Manufacturing Technology. 2009

10. INJECTION MOLDLING MOLDS, Monroe, Engineers Edges, 2013.

11. KRIMPENIS A, et al. Assessment of sculptured surface milling strategies using design of experiments. International Journal of Advanced Manufacturing Technology. 2005.

12. M. HÄNSEL e T. HARNAU. Tool life and tool quality - a summary of the activities of the ICFG subgroup tool life. Thyssen Krupp Presta AG. 2010

13. MACHINABILITY, Dhahran, King Fahd University of Petroleum and Minerals, 2009.

14. MIN, S. et al. Variation in machinability of single crystal materials in micromachining. Annals of CIRP. 2006.

15. P. J. Agnew. What to consider when evaluating solid carbide end mill machining. Mitsubishi Materials. 2004

16. QEHAJA, N.E. et al. Machinability of metals, methods and pratical application. Annals of DAAAM for 2012 \& Proceedings of the 23th International DAAAM Symposium. 2004

17. SANTOS, A. et al, Usinagem em altíssimas velocidades : como os conceitos HSM/HSC podem revolucionar a indústria a indústria metal-mecânica, São Paulo, Erica, 2003.

18. SIMONEAU, A. et al. Surface defects during microcutting. International Journal of Machine Tools and Manufacture.2006

19. TORRES, C.D. at el. Analyzing the performace of diamond-coated micro end mills internacional. International Journal of Machine Tools \& Manufacture.2009. 